\title{
A Mazur-Ulam problem in non-Archimedean $n$-normed spaces
}

\author{
Hahng-Yun Chu and Se-Hyun Ku*
}

"Correspondence: shku@cnu.ac.kr Department of Mathematics,

Chungnam National University, 79, Daehangno, Yuseong-Gu, Daejeon 305-764, Republic of Korea

\begin{abstract}
In this article, we study the notions of $n$-isometries in non-Archimedean $n$-normed spaces over linear ordered non-Archimedean fields and prove the Mazur-Ulam theorem in the spaces. Furthermore, we obtain some properties for $n$-isometries in non-Archimedean $n$-normed spaces.
\end{abstract}

MSC: Primary 46B20; secondary 51M25; 46S10

Keywords: Mazur-Ulam theorem; $n$-isometry; non-Archimedean n-normed space

\section{Introduction}

Let $X$ and $Y$ be metric spaces with metrics $d_{X}$ and $d_{Y}$, respectively. A map $f: X \rightarrow Y$ is called an isometry if $d_{Y}(f(x), f(y))=d_{X}(x, y)$ for every $x, y \in X$. Mazur and Ulam [1] treated the theory of isometry for the first time. They have proved the following theorem.

Mazur-Ulam theorem Let $f$ be an isometric transformation from a real normed vector space $X$ onto a real normed vector space $Y$ with $f(0)=0$. Then $f$ is linear.

It was natural to ask if the result holds without the onto assumption. Having asked this natural question, Baker [2] answered that every isometry of a real normed linear space into a strictly convex real normed linear space is affine. The Mazur-Ulam theorem has been widely studied by [3-12].

Chu et al. [13] have defined the notion of a 2-isometry which is suitable to represent the concept of an area-preserving mapping in linear 2-normed spaces. In [14], Chu proved that the Mazur-Ulam theorem holds in linear 2-normed spaces under the condition that a 2-isometry preserves collinearity. Chu et al. [15] discussed characteristics of 2-isometries. In [16], Amyari and Sadeghi proved the Mazur-Ulam theorem in non-Archimedean 2normed spaces under the condition of strict convexity. Recently, Choy et al. [17] proved the theorem on non-Archimedean 2-normed spaces over linear ordered non-Archimedean fields without the strict convexity assumption.

Misiak $[18,19]$ defined the concept of an $n$-normed space and investigated the space. Park and Rassias [20] investigated the stability of linear $n$-isometries in a linear $n$-normed Banach module. Chu et al. [21], in linear $n$-normed spaces, defined the concept of an $n$ isometry that is suitable to represent the notion of a volume-preserving mapping. In [22], Chu et al. generalized the Mazur-Ulam theorem to $n$-normed spaces.

In this paper, without the condition of strict convexity, we prove the (additive) MazurUlam theorem on non-Archimedean $n$-normed spaces. Firstly, we assert that an $n$ -

C) 2013 Chu and Ku: licensee Springer. This is an Open Access article distributed under the terms of the Creative Commons Attribution License (http://creativecommons.org/licenses/by/2.0), which permits unrestricted use, distribution, and reproduction in any medium, provided the original work is properly cited. 
isometry $f$ from a non-Archimedean space to a non-Archimedean space preserves the midpoint of a segment under some condition about the set of all elements of a valued field whose valuations are 1. Using the above result, we show the Mazur-Ulam theorem on nonArchimedean $n$-normed spaces over linear ordered non-Archimedean fields. In addition, we prove that the barycenter of a triangle in the non-Archimedean $n$-normed spaces is $f$ invariant under different conditions from those referred in previous statements. And then we also prove the (second type) Mazur-Ulam theorem in non-Archimedean $n$-normed spaces under some different conditions.

\section{The Mazur-Ulam theorem I in non-Archimedean $\boldsymbol{n}$-normed spaces}

In this section, we introduce a non-Archimedean $n$-normed space which is a kind of generalization of a non-Archimedean 2-normed space, and we show the (additive) Mazur-Ulam theorem for an $n$-isometry $f$ defined on a non-Archimedean $n$-normed space, that is, $f(x)-f(0)$ is additive. Firstly, we consider some definitions and lemmas which are needed to prove the theorem.

Recall that a non-Archimedean (or ultrametric) valuation is given by a map $|\cdot|$ from a field $\mathcal{K}$ into $[0, \infty)$ such that for all $r, s \in \mathcal{K}$,

(i) $|r|=0$ if and only if $r=0$;

(ii) $|r s|=|r||s|$;

(iii) $|r+s| \leq \max \{|r|,|s|\}$.

If every element of $\mathcal{K}$ carries a valuation, then a field $\mathcal{K}$ is called a valued field; for convenience, we simply call it a field. It is obvious that $|1|=|-1|=1$ and $|n| \leq 1$ for all $n \in \mathbb{N}$. A trivial example of a non-Archimedean valuation is the map $|\cdot|$ taking everything but 0 into 1 and $|0|=0$ (see [23]).

Let $\mathcal{X}$ be a vector space over a valued field $\mathcal{K}$. A non-Archimedean norm is a function $\|\cdot\|: \mathcal{X} \rightarrow[0, \infty)$ such that for all $r \in \mathcal{K}$ and $x, y \in \mathcal{X}$,

(i) $\|x\|=0$ if and only if $x=0$;

(ii) $\|r x\|=|r|\|x\|$;

(iii) the strong triangle inequality

$$
\|x+y\| \leq \max \{\|x\|,\|y\|\} .
$$

Then we say $(\mathcal{X},\|\cdot\|)$ is a non-Archimedean space.

Definition 2.1 Let $\mathcal{X}$ be a vector space with the dimension greater than $n-1$ over a valued field $\mathcal{K}$ with a non-Archimedean valuation $|\cdot|$. A function $\|\cdot, \ldots, \cdot\|: \mathcal{X} \times \cdots \times \mathcal{X} \rightarrow[0, \infty)$ is said to be a non-Archimedean n-norm if

(i) $\left\|x_{1}, \ldots, x_{n}\right\|=0 \Leftrightarrow x_{1}, \ldots, x_{n}$ are linearly dependent;

(ii) $\left\|x_{1}, \ldots, x_{n}\right\|=\left\|x_{j_{1}}, \ldots, x_{j_{n}}\right\|$ for every permutation $\left(j_{1}, \ldots, j_{n}\right)$ of $(1, \ldots, n)$;

(iii) $\left\|\alpha x_{1}, \ldots, x_{n}\right\|=|\alpha|\left\|x_{1}, \ldots, x_{n}\right\|$;

(iv) $\left\|x+y, x_{2}, \ldots, x_{n}\right\| \leq \max \left\{\left\|x, x_{2}, \ldots, x_{n}\right\|,\left\|y, x_{2}, \ldots, x_{n}\right\|\right\}$

for all $\alpha \in \mathcal{K}$ and all $x, y, x_{1}, \ldots, x_{n} \in \mathcal{X}$. Then $(\mathcal{X},\|\cdot, \ldots, \cdot\|)$ is called a non-Archimedean $n$-normed space.

From now on, let $\mathcal{X}$ and $\mathcal{Y}$ be non-Archimedean $n$-normed spaces over a linear ordered non-Archimedean field $\mathcal{K}$. 
Definition 2.2 Let $\mathcal{X}$ and $\mathcal{Y}$ be non-Archimedean $n$-normed spaces and $f: \mathcal{X} \rightarrow \mathcal{Y}$ be a mapping. We call $f$ an $n$-isometry if

$$
\left\|x_{1}-x_{0}, \ldots, x_{n}-x_{0}\right\|=\left\|f\left(x_{1}\right)-f\left(x_{0}\right), \ldots, f\left(x_{n}\right)-f\left(x_{0}\right)\right\|
$$

for all $x_{0}, x_{1}, \ldots, x_{n} \in \mathcal{X}$.

Definition 2.3 The points $x_{0}, x_{1}, \ldots, x_{n}$ of a non-Archimedean $n$-normed space $\mathcal{X}$ are said to be $n$-collinear if for every $i,\left\{x_{j}-x_{i} \mid 0 \leq j \neq i \leq n\right\}$ is linearly dependent.

The points $x_{0}, x_{1}$ and $x_{2}$ of a non-Archimedean $n$-normed space $\mathcal{X}$ are said to be 2collinear if and only if $x_{2}-x_{0}=t\left(x_{1}-x_{0}\right)$ for some element $t$ of the non-Archimedean field $\mathcal{K}$. We denote the set of all elements of $\mathcal{K}$ whose valuations are 1 by $\mathcal{C}$, that is, $\mathcal{C}=$ $\{\gamma \in \mathcal{K}:|\gamma|=1\}$.

Lemma 2.4 Let $x_{i}$ be an element of a non-Archimedean n-normed space $\mathcal{X}$ for every $i \in$ $\{1, \ldots, n\}$ and $\gamma \in \mathcal{K}$. Then

$$
\left\|x_{1}, \ldots, x_{i}, \ldots, x_{j}, \ldots, x_{n}\right\|=\left\|x_{1}, \ldots, x_{i}, \ldots, x_{j}+\gamma x_{i}, \ldots, x_{n}\right\|
$$

for all $1 \leq i \neq j \leq n$.

Proof By Definition 2.1, we have

$$
\begin{aligned}
\left\|x_{1}, \ldots, x_{i}, \ldots, x_{j}+\gamma x_{i}, \ldots, x_{n}\right\| \\
\quad \leq \max \left\{\left\|x_{1}, \ldots, x_{i}, \ldots, x_{j}, \ldots, x_{n}\right\|,|\gamma|\left\|x_{1}, \ldots, x_{i}, \ldots, x_{i}, \ldots, x_{n}\right\|\right\} \\
\quad=\max \left\{\left\|x_{1}, \ldots, x_{i}, \ldots, x_{j}, \ldots, x_{n}\right\|, 0\right\} \\
\quad=\left\|x_{1}, \ldots, x_{i}, \ldots, x_{j}, \ldots, x_{n}\right\| .
\end{aligned}
$$

One can easily prove the converse using similar methods. This completes the proof.

Remark 2.5 Let $\mathcal{X}, \mathcal{Y}$ be non-Archimedean $n$-normed spaces over a linear ordered nonArchimedean field $\mathcal{K}$ and let $f: \mathcal{X} \rightarrow \mathcal{Y}$ be an $n$-isometry. One can show that the $n$ isometry $f$ from $\mathcal{X}$ to $\mathcal{Y}$ preserves the 2-collinearity using a similar method to that in [22, Lemma 3.2].

The midpoint of a segment with endpoints $x$ and $y$ in the non-Archimedean $n$-normed space $\mathcal{X}$ is defined by the point $\frac{x+y}{2}$.

Now, we prove the Mazur-Ulam theorem on non-Archimedean $n$-normed spaces. In the first step, we prove the following lemma. And then, using the lemma, we show that an $n$-isometry $f$ from a non-Archimedean $n$-normed space $\mathcal{X}$ to a non-Archimedean $n$ normed space $\mathcal{Y}$ preserves the midpoint of a segment, i.e., the $f$-image of the midpoint of a segment in $\mathcal{X}$ is also the midpoint of a corresponding segment in $\mathcal{Y}$.

Lemma 2.6 Let $\mathcal{X}$ be a non-Archimedean n-normed space over a linear ordered nonArchimedean field $\mathcal{K}$ with $\mathcal{C}=\left\{2^{n} \mid n \in \mathbb{Z}\right\}$ and let $x_{0}, x_{1} \in \mathcal{X}$ with $x_{0} \neq x_{1}$. Then $u:=\frac{x_{0}+x_{1}}{2}$ is 
the unique member of $\mathcal{X}$ satisfying

$$
\begin{aligned}
& \left\|x_{0}-x_{1}, x_{0}-x_{2}, x_{0}-x_{3}, \ldots, x_{0}-x_{n}\right\| \\
& =\left\|x_{0}-u, x_{0}-x_{2}, x_{0}-x_{3}, \ldots, x_{0}-x_{n}\right\| \\
& =\left\|x_{1}-u, x_{1}-x_{2}, x_{1}-x_{3}, \ldots, x_{1}-x_{n}\right\|
\end{aligned}
$$

for some $x_{2}, \ldots, x_{n} \in \mathcal{X}$ with $\left\|x_{0}-x_{1}, x_{0}-x_{2}, \ldots, x_{0}-x_{n}\right\| \neq 0$ and $u, x_{0}, x_{1}$ are 2 -collinear.

Proof Let $u:=\frac{x_{0}+x_{1}}{2}$. From the assumption for the dimension of $\mathcal{X}$, there exist $n-1$ elements $x_{2}, \ldots, x_{n}$ in $\mathcal{X}$ such that $\left\|x_{0}-x_{1}, x_{0}-x_{2}, \ldots, x_{0}-x_{n}\right\| \neq 0$. One can easily prove that $u$ satisfies the above equations and conditions. It suffices to show the uniqueness for $u$. Assume that there is another $v$ satisfying

$$
\begin{aligned}
& \left\|x_{0}-x_{1}, x_{0}-x_{2}, x_{0}-x_{3}, \ldots, x_{0}-x_{n}\right\| \\
& =\left\|x_{0}-v, x_{0}-x_{2}, x_{0}-x_{3}, \ldots, x_{0}-x_{n}\right\| \\
& =\left\|x_{1}-v, x_{1}-x_{2}, x_{1}-x_{3}, \ldots, x_{1}-x_{n}\right\|
\end{aligned}
$$

for some elements $x_{2}, \ldots, x_{n}$ of $\mathcal{X}$ with $\left\|x_{0}-x_{1}, x_{0}-x_{2}, \ldots, x_{0}-x_{n}\right\| \neq 0$ and $v, x_{0}, x_{1}$ are 2 -collinear. Since $v, x_{0}, x_{1}$ are 2-collinear, $v=t x_{0}+(1-t) x_{1}$ for some $t \in \mathcal{K}$. Then we have

$$
\begin{aligned}
& \left\|x_{0}-x_{1}, x_{0}-x_{2}, x_{0}-x_{3}, \ldots, x_{0}-x_{n}\right\| \\
& =\left\|x_{0}-v, x_{0}-x_{2}, x_{0}-x_{3}, \ldots, x_{0}-x_{n}\right\| \\
& =\left\|x_{0}-t x_{0}-(1-t) x_{1}, x_{0}-x_{2}, x_{0}-x_{3}, \ldots, x_{0}-x_{n}\right\| \\
& =|1-t|\left\|x_{0}-x_{1}, x_{0}-x_{2}, x_{0}-x_{3}, \ldots, x_{0}-x_{n}\right\|, \\
& \left\|x_{0}-x_{1}, x_{0}-x_{2}, x_{0}-x_{3}, \ldots, x_{0}-x_{n}\right\| \\
& =\left\|x_{1}-v, x_{1}-x_{2}, x_{1}-x_{3}, \ldots, x_{1}-x_{n}\right\| \\
& =\left\|x_{1}-t x_{0}-(1-t) x_{1}, x_{1}-x_{2}, x_{1}-x_{3}, \ldots, x_{1}-x_{n}\right\| \\
& =|t|\left\|x_{0}-x_{1}, x_{1}-x_{2}, x_{1}-x_{3}, \ldots, x_{1}-x_{n}\right\| \\
& =|t|\left\|x_{0}-x_{1}, x_{0}-x_{2}, x_{0}-x_{3}, \ldots, x_{0}-x_{n}\right\| .
\end{aligned}
$$

Since $\left\|x_{0}-x_{1}, x_{0}-x_{2}, \ldots, x_{0}-x_{n}\right\| \neq 0$, we have two equations $|1-t|=1$ and $|t|=1$. So, there are two integers $k_{1}, k_{2}$ such that $1-t=2^{k_{1}}, t=2^{k_{2}}$. Since $2^{k_{1}}+2^{k_{2}}=1, k_{i}<0$ for all $i=1,2$. Thus, we may assume that $1-t=2^{-n_{1}}, t=2^{-n_{2}}$ and $n_{1} \geq n_{2} \in \mathbb{N}$ without loss of generality. If $n_{1} \geqslant n_{2}$, then $1=2^{-n_{1}}+2^{-n_{2}}=2^{-n_{1}}\left(1+2^{n_{1}-n_{2}}\right)$, that is, $2^{n_{1}}=1+2^{n_{1}-n_{2}}$. This is a contradiction because the left-hand side of the equation is a multiple of 2 but the right-hand side of the equation is not. Thus, $n_{1}=n_{2}=1$ and hence $v=\frac{1}{2} x_{0}+\frac{1}{2} x_{1}=u$.

Theorem 2.7 Let $\mathcal{X}, \mathcal{Y}$ be non-Archimedean n-normed spaces over a linear ordered nonArchimedean field $\mathcal{K}$ with $\mathcal{C}=\left\{2^{n} \mid n \in \mathbb{Z}\right\}$ and $f: \mathcal{X} \rightarrow \mathcal{Y}$ be an n-isometry. Then the midpoint of a segment is $f$-invariant, i.e., for every $x_{0}, x_{1} \in \mathcal{X}$ with $x_{0} \neq x_{1}, f\left(\frac{x_{0}+x_{1}}{2}\right)$ is also the midpoint of a segment with endpoints $f\left(x_{0}\right)$ and $f\left(x_{1}\right)$ in $\mathcal{Y}$. 
Proof Let $x_{0}, x_{1} \in \mathcal{X}$ with $x_{0} \neq x_{1}$. Since the dimension of $\mathcal{X}$ is greater than $n-1$, there exist $n-1$ elements $x_{2}, \ldots, x_{n}$ of $\mathcal{X}$ satisfying $\left\|x_{0}-x_{1}, x_{0}-x_{2}, \ldots, x_{0}-x_{n}\right\| \neq 0$. Since $x_{0}, x_{1}$ and their midpoint $\frac{x_{0}+x_{1}}{2}$ are 2-collinear in $\mathcal{X}, f\left(x_{0}\right), f\left(x_{1}\right), f\left(\frac{x_{0}+x_{1}}{2}\right)$ are also 2-collinear in $\mathcal{Y}$ by Remark 2.5. Since $f$ is an $n$-isometry, we have the following:

$$
\begin{aligned}
& \left\|f\left(x_{0}\right)-f\left(\frac{x_{0}+x_{1}}{2}\right), f\left(x_{0}\right)-f\left(x_{2}\right), \ldots, f\left(x_{0}\right)-f\left(x_{n}\right)\right\| \\
& \quad=\left\|x_{0}-\frac{x_{0}+x_{1}}{2}, x_{0}-x_{2}, \ldots, x_{0}-x_{n}\right\| \\
& \quad=\left|\frac{1}{2}\right|\left\|x_{0}-x_{1}, x_{0}-x_{2}, \ldots, x_{0}-x_{n}\right\| \\
& \quad=\left\|f\left(x_{0}\right)-f\left(x_{1}\right), f\left(x_{0}\right)-f\left(x_{2}\right), \ldots, f\left(x_{0}\right)-f\left(x_{n}\right)\right\|, \\
& \left\|f\left(x_{1}\right)-f\left(\frac{x_{0}+x_{1}}{2}\right), f\left(x_{1}\right)-f\left(x_{2}\right), \ldots, f\left(x_{1}\right)-f\left(x_{n}\right)\right\| \\
& \quad=\left\|x_{1}-\frac{x_{0}+x_{1}}{2}, x_{1}-x_{2}, \ldots, x_{1}-x_{n}\right\| \\
& =\left|\frac{1}{2}\right|\left\|x_{1}-x_{0}, x_{1}-x_{2}, \ldots, x_{1}-x_{n}\right\| \\
& =\left\|f\left(x_{0}\right)-f\left(x_{1}\right), f\left(x_{0}\right)-f\left(x_{2}\right), \ldots, f\left(x_{0}\right)-f\left(x_{n}\right)\right\| .
\end{aligned}
$$

By Lemma 2.6, we obtain that $f\left(\frac{x_{0}+x_{1}}{2}\right)=\frac{f\left(x_{0}\right)+f\left(x_{1}\right)}{2}$ for all $x_{0}, x_{1} \in \mathcal{X}$ with $x_{0} \neq x_{1}$. This completes the proof.

Lemma 2.8 Let $\mathcal{X}$ and $\mathcal{Y}$ be non-Archimedean n-normed spaces over a linear ordered non-Archimedean field $\mathcal{K}$ and $f: \mathcal{X} \rightarrow \mathcal{Y}$ be an n-isometry. Then the following conditions are equivalent.

(i) The n-isometry $f$ preserves the midpoint of a segment in $\mathcal{X}$, i.e., $f\left(\frac{x_{0}+x_{1}}{2}\right)=\frac{f\left(x_{0}\right)+f\left(x_{1}\right)}{2}$ for all $x_{0}, x_{1} \in \mathcal{X}$ with $x_{0} \neq x_{1}$;

(ii) The n-isometry $f$ preserves the barycenter of a triangle in $\mathcal{X}$, i.e., $f\left(\frac{x_{0}+x_{1}+x_{2}}{3}\right)=$ $\frac{f\left(x_{0}\right)+f\left(x_{1}\right)+f\left(x_{2}\right)}{3}$ for all $x_{0}, x_{1}, x_{2} \in \mathcal{X}$ satisfying that $x_{0}, x_{1}, x_{2}$ are not 2-collinear.

Proof Assume that the $n$-isometry $f$ preserves the barycenter of a triangle in $\mathcal{X}$. Let $x_{0}$, $x_{1}$ be in $\mathcal{X}$ with $x_{0} \neq x_{1}$. Since the $n$-isometry $f$ preserves the 2 -collinearity, $f\left(x_{0}\right), f\left(\frac{x_{0}+x_{1}}{2}\right)$, $f\left(x_{1}\right)$ are 2-collinear. So,

$$
f\left(\frac{x_{0}+x_{1}}{2}\right)-f\left(x_{0}\right)=s\left(f\left(x_{1}\right)-f\left(x_{0}\right)\right)
$$

for some element $s$ of $\mathcal{K}$. By the hypothesis for the dimension of $\mathcal{X}$, we can choose the element $x_{2}$ of $\mathcal{X}$ satisfying that $x_{0}, x_{1}$ and $x_{2}$ are not 2-collinear. Since $x_{2}, \frac{x_{0}+x_{1}+x_{2}}{3}, \frac{x_{0}+x_{1}}{2}$ are 2-collinear, we have that $f\left(x_{2}\right), f\left(\frac{x_{0}+x_{1}+x_{2}}{3}\right), f\left(\frac{x_{0}+x_{1}}{2}\right)$ are also 2-collinear by Remark 2.5. So, we obtain that

$$
f\left(\frac{x_{0}+x_{1}+x_{2}}{3}\right)-f\left(x_{2}\right)=t\left(f\left(\frac{x_{0}+x_{1}}{2}\right)-f\left(x_{2}\right)\right)
$$


for some element $t$ of the non-Archimedean field $\mathcal{K}$. By the equations (2.1), (2.2) and the barycenter-preserving property for the $n$-isometry $f$, we have

$$
\frac{f\left(x_{0}\right)+f\left(x_{1}\right)+f\left(x_{2}\right)}{3}-f\left(x_{2}\right)=t\left(f\left(x_{0}\right)+s f\left(x_{1}\right)-s f\left(x_{0}\right)-f\left(x_{2}\right)\right) .
$$

Thus, we get

$$
\frac{f\left(x_{0}\right)+f\left(x_{1}\right)-2 f\left(x_{2}\right)}{3}=t(1-s) f\left(x_{0}\right)+t s f\left(x_{1}\right)-t f\left(x_{2}\right) .
$$

So, we have the following equation:

$$
\frac{2}{3}\left(f\left(x_{0}\right)-f\left(x_{2}\right)\right)-\frac{1}{3}\left(f\left(x_{0}\right)-f\left(x_{1}\right)\right)=t\left(f\left(x_{0}\right)-f\left(x_{2}\right)\right)-t s\left(f\left(x_{0}\right)-f\left(x_{1}\right)\right) .
$$

By a calculation, we obtain

$$
\left(\frac{2}{3}-t\right)\left(f\left(x_{0}\right)-f\left(x_{2}\right)\right)+\left(-\frac{1}{3}+t s\right)\left(f\left(x_{0}\right)-f\left(x_{1}\right)\right)=0 .
$$

Since $x_{0}, x_{1}, x_{2}$ are not 2-collinear, $x_{0}-x_{1}, x_{0}-x_{2}$ are linearly independent. Since $\operatorname{dim} \mathcal{X} \geq$ $n$, there are $x_{3}, \ldots, x_{n} \in \mathcal{X}$ such that $\left\|x_{0}-x_{1}, x_{0}-x_{2}, x_{0}-x_{3}, \ldots, x_{0}-x_{n}\right\| \neq 0$. Since $f$ is an $n$-isometry,

$$
\begin{aligned}
& \left\|f\left(x_{0}\right)-f\left(x_{1}\right), f\left(x_{0}\right)-f\left(x_{2}\right), f\left(x_{0}\right)-f\left(x_{3}\right), \ldots, f\left(x_{0}\right)-f\left(x_{n}\right)\right\| \\
& \quad=\left\|x_{0}-x_{1}, x_{0}-x_{2}, x_{0}-x_{3}, \ldots, x_{0}-x_{n}\right\| \neq 0 .
\end{aligned}
$$

So, $f\left(x_{0}\right)-f\left(x_{1}\right)$ and $f\left(x_{0}\right)-f\left(x_{2}\right)$ are linearly independent. Hence, from equation (2.3), we have $\frac{2}{3}-t=0$ and $-\frac{1}{3}+t s=0$, i.e., we obtain $t=\frac{2}{3}, s=\frac{1}{2}$, which imply the equation

$$
f\left(\frac{x_{0}+x_{1}}{2}\right)=\frac{f\left(x_{0}\right)+f\left(x_{1}\right)}{2}
$$

for all $x_{0}, x_{1} \in \mathcal{X}$ with $x_{0} \neq x_{1}$.

Conversely, (i) trivially implies (ii). This completes the proof of this lemma.

Remark 2.9 One can prove that the above lemma also holds in the case of linear $n$ normed spaces.

Theorem 2.10 Let $\mathcal{X}$ and $\mathcal{Y}$ be non-Archimedean n-normed spaces over a linear ordered non-Archimedean field $\mathcal{K}$ with $\mathcal{C}=\left\{2^{n} \mid n \in \mathbb{Z}\right\}$. If $f: \mathcal{X} \rightarrow \mathcal{Y}$ is an n-isometry, then $f(x)-$ $f(0)$ is additive.

Proof Let $g(x):=f(x)-f(0)$. Then it is clear that $g(0)=0$ and $g$ is also an $n$-isometry.

From Theorem 2.7, for $x_{0}, x_{1} \in \mathcal{X}\left(x_{0} \neq x_{1}\right)$, we have

$$
g\left(\frac{x_{0}+x_{1}}{2}\right)=\frac{g\left(x_{0}\right)+g\left(x_{1}\right)}{2} .
$$

Since $g(0)=0$, we obtain that $g$ is additive which completes the proof. 


\section{The Mazur-Ulam theorem II in non-Archimedean $\boldsymbol{n}$-normed spaces}

In this section, under different conditions from those previously referred in Theorem 2.10, we also prove the (second type) Mazur-Ulam theorem on a non-Archimedean $n$-normed space. Firstly, we show that an $n$-isometry $f$ from a non-Archimedean $n$-normed space $\mathcal{X}$ to a non-Archimedean $n$-normed space $\mathcal{Y}$ preserves the barycenter of a triangle, i.e., the $f$-image of the barycenter of a triangle is also the barycenter of a corresponding triangle. Then, using Lemma 2.8, we also prove the Mazur-Ulam theorem (a non-Archimedean $n$-normed space version) under some different conditions.

Lemma 3.1 Let $\mathcal{X}$ be a non-Archimedean n-normed space over a linear ordered nonArchimedean field $\mathcal{K}$ with $\mathcal{C}=\left\{3^{n} \mid n \in \mathbb{Z}\right\}$ and let $x_{0}, x_{1}, x_{2}$ be elements of $\mathcal{X}$ such that $x_{0}, x_{1}, x_{2}$ are not 2-collinear. Then $u:=\frac{x_{0}+x_{1}+x_{2}}{3}$ is the unique member of $\mathcal{X}$ satisfying

$$
\begin{aligned}
\| x_{0} & -x_{1}, x_{0}-x_{2}, x_{0}-x_{3}, \ldots, x_{0}-x_{n} \| \\
& =\left\|x_{0}-x_{1}, x_{0}-u, x_{0}-x_{3}, \ldots, x_{0}-x_{n}\right\| \\
& =\left\|x_{1}-x_{2}, x_{1}-u, x_{1}-x_{3}, \ldots, x_{1}-x_{n}\right\| \\
& =\left\|x_{2}-x_{0}, x_{2}-u, x_{2}-x_{3}, \ldots, x_{2}-x_{n}\right\|
\end{aligned}
$$

for some $x_{3}, \ldots, x_{n} \in \mathcal{X}$ with $\left\|x_{0}-x_{1}, x_{0}-x_{2}, x_{0}-x_{3}, \ldots, x_{0}-x_{n}\right\| \neq 0$ and $u$ is an interior point of $\triangle_{x_{0} x_{1} x_{2}}$.

Proof Let $u:=\frac{x_{0}+x_{1}+x_{2}}{3}$. Thus $u$ is an interior point of $\triangle_{x_{0} x_{1} x_{2}}$. Since $\operatorname{dim} \mathcal{X}>n-1$, there are $n-2$ elements $x_{3}, \ldots, x_{n}$ of $\mathcal{X}$ such that $\left\|x_{0}-x_{1}, x_{0}-x_{2}, x_{0}-x_{3}, \ldots, x_{0}-x_{n}\right\| \neq 0$. Applying Lemma 2.4, we have that

$$
\begin{aligned}
& \left\|x_{0}-x_{1}, x_{0}-u, x_{0}-x_{3}, \ldots, x_{0}-x_{n}\right\| \\
& \quad=\left\|x_{0}-x_{1}, x_{0}-\frac{x_{0}+x_{1}+x_{2}}{3}, x_{0}-x_{3}, \ldots, x_{0}-x_{n}\right\| \\
& \quad=\left|\frac{1}{3}\right|\left\|x_{0}-x_{1}, x_{0}-x_{1}+x_{0}-x_{2}, x_{0}-x_{3}, \ldots, x_{0}-x_{n}\right\| \\
& =\left\|x_{0}-x_{1}, x_{0}-x_{2}, x_{0}-x_{3}, \ldots, x_{0}-x_{n}\right\| .
\end{aligned}
$$

And we can also obtain that

$$
\begin{aligned}
\left\|x_{0}-x_{1}, x_{0}-x_{2}, x_{0}-x_{3}, \ldots, x_{0}-x_{n}\right\| \\
=\left\|x_{1}-x_{2}, x_{1}-u, x_{1}-x_{3}, \ldots, x_{1}-x_{n}\right\| \\
=\left\|x_{2}-x_{0}, x_{2}-u, x_{2}-x_{3}, \ldots, x_{2}-x_{n}\right\| .
\end{aligned}
$$

For the proof of uniqueness, let $v$ be another interior point of $\triangle_{x_{0} x_{1} x_{2}}$ satisfying

$$
\begin{aligned}
& \left\|x_{0}-x_{1}, x_{0}-x_{2}, x_{0}-x_{3}, \ldots, x_{0}-x_{n}\right\| \\
& \quad=\left\|x_{0}-x_{1}, x_{0}-v, x_{0}-x_{3}, \ldots, x_{0}-x_{n}\right\|
\end{aligned}
$$




$$
\begin{aligned}
& =\left\|x_{1}-x_{2}, x_{1}-v, x_{1}-x_{3}, \ldots, x_{1}-x_{n}\right\| \\
& =\left\|x_{2}-x_{0}, x_{2}-v, x_{2}-x_{3}, \ldots, x_{2}-x_{n}\right\|
\end{aligned}
$$

with $\left\|x_{0}-x_{1}, x_{0}-x_{2}, x_{0}-x_{3}, \ldots, x_{0}-x_{n}\right\| \neq 0$. Since $v$ is an element of the set $\left\{t_{0} x_{0}+t_{1} x_{1}+\right.$ $t_{2} x_{2} \mid t_{0}+t_{1}+t_{2}=1, t_{i} \in \mathcal{K}, t_{i}>0$ for all $\left.i\right\}$, there are elements $s_{0}, s_{1}, s_{2}$ of $\mathcal{K}$ with $s_{0}+s_{1}+s_{2}=1$, $s_{i}>0$ such that $v=s_{0} x_{0}+s_{1} x_{1}+s_{2} x_{2}$. Then we have

$$
\begin{aligned}
\left\|x_{0}-x_{1}, x_{0}-x_{2}, x_{0}-x_{3}, \ldots, x_{0}-x_{n}\right\| \\
\quad=\left\|x_{0}-x_{1}, x_{0}-v, x_{0}-x_{3}, \ldots, x_{0}-x_{n}\right\| \\
\quad=\left\|x_{0}-x_{1}, x_{0}-s_{0} x_{0}-s_{1} x_{1}-s_{2} x_{2}, x_{0}-x_{3}, \ldots, x_{0}-x_{n}\right\| \\
\quad=\left\|x_{0}-x_{1},\left(s_{0}-1\right) x_{0}+s_{1} x_{1}+\left(1-s_{0}-s_{1}\right) x_{2}, x_{0}-x_{3}, \ldots, x_{0}-x_{n}\right\| \\
=\left\|x_{0}-x_{1},\left(s_{0}+s_{1}-1\right) x_{0}+\left(1-s_{0}-s_{1}\right) x_{2}, x_{0}-x_{3}, \ldots, x_{0}-x_{n}\right\| \\
=\left|s_{0}+s_{1}-1\right|\left\|x_{0}-x_{1}, x_{0}-x_{2}, x_{0}-x_{3}, \ldots, x_{0}-x_{n}\right\| \\
\quad=\left|s_{2}\right|\left\|x_{0}-x_{1}, x_{0}-x_{2}, x_{0}-x_{3}, \ldots, x_{0}-x_{n}\right\|
\end{aligned}
$$

and hence $\left|s_{2}\right|=1$ since $\left\|x_{0}-x_{1}, x_{0}-x_{2}, x_{0}-x_{3}, \ldots, x_{0}-x_{n}\right\| \neq 0$. Similarly, we obtain $\left|s_{0}\right|=$ $\left|s_{1}\right|=1$. By the hypothesis of $\mathcal{C}$, there are integers $k_{0}, k_{1}, k_{2}$ such that $s_{0}=3^{k_{1}}, s_{1}=3^{k_{2}}$, $s_{2}=3^{k_{2}}$. Since $s_{0}+s_{1}+s_{2}=1$, every $k_{i}$ is less than 0 . So, one may let $s_{0}=3^{-n_{0}}, s_{1}=3^{-n_{1}}$, $s_{2}=3^{-n_{2}}$ and $n_{0} \geq n_{1} \geq n_{2} \in \mathbb{N}$. Assume that one of the above inequalities holds. Then $1=s_{0}+s_{1}+s_{2}=3^{-n_{0}}\left(1+3^{n_{0}-n_{1}}+3^{n_{0}-n_{2}}\right)$, i.e., $3^{n_{0}}=1+3^{n_{0}-n_{1}}+3^{n_{0}-n_{2}}$. This is a contradiction, because the left-hand side is a multiple of 3 whereas the right-hand side is not. Thus, $n_{0}=n_{1}=n_{2}$. Consequently, $s_{0}=s_{1}=s_{2}=\frac{1}{3}$. This means that $u$ is unique.

Theorem 3.2 Let $\mathcal{X}, \mathcal{Y}$ be non-Archimedean n-normed spaces over a linear ordered nonArchimedean field $\mathcal{K}$ with $\mathcal{C}=\left\{3^{n} \mid n \in \mathbb{Z}\right\}$ and $f: \mathcal{X} \rightarrow \mathcal{Y}$ be an interior-preserving $n$ isometry. Then the barycenter of a triangle is $f$-invariant.

Proof Let $x_{0}, x_{1}$ and $x_{2}$ be elements of $\mathcal{X}$ satisfying that $x_{0}, x_{1}$ and $x_{2}$ are not 2-collinear. It is obvious that the barycenter $\frac{x_{0}+x_{1}+x_{2}}{3}$ of a triangle $\triangle_{x_{0} x_{1} x_{2}}$ is an interior point of the triangle. By the assumption, $f\left(\frac{x_{0}+x_{1}+x_{2}}{3}\right)$ is also the interior point of a triangle $\triangle_{f\left(x_{0}\right) f\left(x_{1}\right) f\left(x_{2}\right)}$. Since $\operatorname{dim} \mathcal{X}>n-1$, there exist $n-2$ elements $x_{3}, \ldots, x_{n}$ in $\mathcal{X}$ such that $\| x_{0}-x_{1}, x_{0}-x_{2}, x_{0}-$ $x_{3}, \ldots, x_{0}-x_{n} \|$ is not zero. Since $f$ is an $n$-isometry, we have

$$
\begin{aligned}
& \left\|f\left(x_{0}\right)-f\left(x_{1}\right), f\left(x_{0}\right)-f\left(\frac{x_{0}+x_{1}+x_{2}}{3}\right), f\left(x_{0}\right)-f\left(x_{3}\right), \ldots, f\left(x_{0}\right)-f\left(x_{n}\right)\right\| \\
& \quad=\left\|x_{0}-x_{1}, x_{0}-\frac{x_{0}+x_{1}+x_{2}}{3}, x_{0}-x_{3}, \ldots, x_{0}-x_{n}\right\| \\
& \quad=\left|\frac{1}{3}\right|\left\|x_{0}-x_{1}, x_{0}-x_{1}+x_{0}-x_{2}, x_{0}-x_{3}, \ldots, x_{0}-x_{n}\right\| \\
& \quad=\left\|x_{0}-x_{1}, x_{0}-x_{2}, x_{0}-x_{3}, \ldots, x_{0}-x_{n}\right\| \\
& \quad=\left\|f\left(x_{0}\right)-f\left(x_{1}\right), f\left(x_{0}\right)-f\left(x_{2}\right), f\left(x_{0}\right)-f\left(x_{3}\right), \ldots, f\left(x_{0}\right)-f\left(x_{n}\right)\right\| .
\end{aligned}
$$


Similarly, we obtain

$$
\begin{aligned}
& \left\|f\left(x_{1}\right)-f\left(x_{2}\right), f\left(x_{1}\right)-f\left(\frac{x_{0}+x_{1}+x_{2}}{3}\right), f\left(x_{1}\right)-f\left(x_{3}\right), \ldots, f\left(x_{1}\right)-f\left(x_{n}\right)\right\| \\
& \quad=\left\|f\left(x_{2}\right)-f\left(x_{1}\right), f\left(x_{2}\right)-f\left(\frac{x_{0}+x_{1}+x_{2}}{3}\right), f\left(x_{2}\right)-f\left(x_{3}\right), \ldots, f\left(x_{2}\right)-f\left(x_{n}\right)\right\| \\
& \quad=\left\|f\left(x_{0}\right)-f\left(x_{1}\right), f\left(x_{0}\right)-f\left(x_{2}\right), f\left(x_{0}\right)-f\left(x_{3}\right), \ldots, f\left(x_{0}\right)-f\left(x_{n}\right)\right\| .
\end{aligned}
$$

From Lemma 3.1, we get

$$
f\left(\frac{x_{0}+x_{1}+x_{2}}{3}\right)=\frac{f\left(x_{0}\right)+f\left(x_{1}\right)+f\left(x_{2}\right)}{3}
$$

for all $x_{0}, x_{1}, x_{2} \in \mathcal{X}$ satisfying that $x_{0}, x_{1}, x_{2}$ are not 2 -collinear.

The next theorem is the Mazur-Ulam theorem II in non-Archimedean $n$-normed spaces over a linear ordered non-Archimedean field $\mathcal{K}$. The assumptions of this theorem are different from that of Theorem 2.10. In particular, in the proof of the theorem, we use the $f$-preserving property for the barycenter of a triangle.

Theorem 3.3 Let $\mathcal{X}$ and $\mathcal{Y}$ be non-Archimedean n-normed spaces over a linear ordered non-Archimedean field $\mathcal{K}$ with $\mathcal{C}=\left\{3^{n} \mid n \in \mathbb{Z}\right\}$. If $f: \mathcal{X} \rightarrow \mathcal{Y}$ is an interior-preserving $n$ isometry, then $f(x)-f(0)$ is additive.

Proof Let $g(x):=f(x)-f(0)$. One can easily check that $g(0)=0$ and $g$ is also an $n$-isometry. Using a similar method in [17, Theorem 2.4], we can easily prove that $g$ is also an interiorpreserving mapping.

Now, let $x_{0}, x_{1}, x_{2}$ be elements of $\mathcal{X}$ satisfying that $x_{0}, x_{1}, x_{2}$ are not 2-collinear. Since $g$ is an interior-preserving $n$-isometry, by Theorem 3.2,

$$
g\left(\frac{x_{0}+x_{1}+x_{2}}{3}\right)=\frac{g\left(x_{0}\right)+g\left(x_{1}\right)+g\left(x_{2}\right)}{3}
$$

for any $x_{0}, x_{1}, x_{2} \in \mathcal{X}$ satisfying that $x_{0}, x_{1}, x_{2}$ are not 2-collinear. Using Lemma 2.8 and the property $g(0)=0$, we obtain that the interior-preserving $n$-isometry $g$ is additive, which completes the proof.

\section{Competing interests}

The authors declare that they have no competing interests.

Authors' contributions

The authors conceived of the study, participated in design and coordination of the manuscript. They drafted the manuscript and participated in the sequence alignment. They also read and approved the final manuscript.

\section{Acknowledgements}

The authors are deeply grateful to the referees whose helped to improve our manuscript. This research was supported by Basic Science Research Program through the National Research Foundation of Korea (NRF) funded by the Ministry of Education, Science and Technology (2010-0013784). 


\section{References}

1. Mazur, S, Ulam, S: Sur les transformationes isométriques d'espaces vectoriels normés. C. R. Math. Acad. Sci. Paris 194, 946-948 (1932)

2. Baker, JA: Isometries in normed spaces. Am. Math. Mon. 78, 655-658 (1971)

3. Cho, YJ, Lin, PCS, Kim, SS, Misiak, A: Theory of 2-Linear Product Spaces. Nova Science Publishers, New York (2001)

4. Freese, RW, Cho, YJ: Isometry conditions in linear 2-normed spaces. Math. Jpn. 35, 985-990 (1990)

5. Freese, RW, Cho, YJ: Geometry of Linear 2-Normed Spaces. Nova Science Publishers, New York (2001)

6. Jian, W: On the generalizations of the Mazur-Ulam isometric theorem. J. Math. Anal. Appl. 263, $510-521$ (2001)

7. Moslehian, MS, Sadeghi, Gh: A Mazur-Ulam theorem in non-Archimedean normed spaces. Nonlinear Anal. 69, 3405-3408 (2008)

8. Rassias, TM: On the A.D. Aleksandrov problem of conservative distances and the Mazur-Ulam theorem. Nonlinear Anal. 47, 2597-2608 (2001)

9. Rassias, TM, Šemrl, P: On the Mazur-Ulam theorem and the Aleksandrov problem for unit distance preserving mappings. Proc. Am. Math. Soc. 118, 919-925 (1993)

10. Rassias, TM, Wagner, P: Volume preserving mappings in the spirit of the Mazur-Ulam theorem. Aequ. Math. 66, 85-89 (2003)

11. Väisälä, J: A proof of the Mazur-Ulam theorem. Am. Math. Mon. 110, 633-635 (2003)

12. Xiang, S: Mappings of conservative distances and the Mazur-Ulam theorem. J. Math. Anal. Appl. 254, 262-274 (2001)

13. Chu, H-Y, Park, C-K, Park, W-K: The Aleksandrov problem in linear 2-normed spaces. J. Math. Anal. Appl. 289, 666-672 (2004)

14. Chu, H-Y: On the Mazur-Ulam problem in linear 2-normed spaces. J. Math. Anal. Appl. 327, 1041-1045 (2007)

15. $\mathrm{Chu}, \mathrm{H}-\mathrm{Y}, \mathrm{Ku}, \mathrm{S}-\mathrm{H}, \mathrm{Kang}, \mathrm{DS}$ : Characterizations on 2-isometries. J. Math. Anal. Appl. 340, 621-628 (2008)

16. Amyari, M, Sadeghi, G: Isometries in non-Archimedean strictly convex and strictly 2-convex 2-normed spaces. In: Nonlinear Analysis and Variational Problems, pp. 13-22. Springer, Berlin (2009)

17. Choy, J, Chu, H-Y, Ku, S-H: Characterizations on Mazur-Ulam theorem. Nonlinear Anal. 72, 1291-1297 (2010)

18. Misiak, A: $n$-inner product spaces. Math. Nachr. 140, 299-319 (1989)

19. Misiak, A: Orthogonality and orthogonormality in $n$-inner product spaces. Math. Nachr. 143, $249-261$ (1989)

20. Park, C-K, Rassias, TM: The N-isometric isomorphisms in linear N-normed $C^{*}$-algebras. Acta Math. Sin. Engl. Ser. 22, 1863-1890 (2006)

21. Chu, H-Y, Lee, K, Park, C-K: On the Aleksandrov problem in linear n-normed spaces. Nonlinear Anal. 59, 1001-1011 (2004)

22. Chu, H-Y, Choi, SK, Kang, DS: Mappings of conservative distances in linear n-normed spaces. Nonlinear Anal. 70 , 1168-1174 (2009)

23. Narici, L, Beckenstein, E: Strange terrain-non-Archimedean spaces. Am. Math. Mon. 88, 667-676 (1981)

doi:10.1186/1029-242X-2013-34

Cite this article as: Chu and Ku: A Mazur-Ulam problem in non-Archimedean n-normed spaces. Journal of Inequalities and Applications 2013 2013:34

\section{Submit your manuscript to a SpringerOpen ${ }^{\ominus}$ journal and benefit from:}

- Convenient online submission

- Rigorous peer review

- Immediate publication on acceptance

- Open access: articles freely available online

- High visibility within the field

- Retaining the copyright to your article 